Ann. Génét. Sél. anim., I975, 7 (4), 379-386.

\title{
COEFFICIENT DE DÉPRESSION ET PANMIXIE
}

\author{
Jacqueline BOUFFETTE et A.-R. BOUFFETTE \\ Université Claude-Bernard, Lyon I, \\ Département de Mathématiques, \\ 43, boulevard du 11-Novembre-1918, \\ 69621 Villeurbanne
}

\section{RÉSUMÉ}

\begin{abstract}
L'étude d'une population panmictique, d'effectif important, est faite dans l'hypothèse de génétique non-mendélienne, où les mécanismes de la consanguinité impliquent des interactions gamétiques entre des sites (ou gènes) actifs ou inactifs (BiÉmont et al., 1974).

Les algèbres génétiques d'ETHERINGToN (1940) permettent de déterminer l'évolution de la probabilité pour qu'un couple de sites gamétiques homologues soit hétéro-actif. Lorsque le nombre de générations augmente, cette probabilité tend en général vers une limite indépendante des zygotes de la génération initiale. Il en est alors de même pour le coefficient de dépression.

Cependant, si les taux de " mutation "sont élevés, la probabilité fluctue entre deux limites distinctes. Cette variation se traduit par un coefficient de dépression limite non constant.

La comparaison entre le coefficient de dépression d'un individu issu de parents quelconques, celui d'un individu issu de parents " demi-frère " et celui d'un individu issu d'un croisement "frèresœur " conduit, lorsqu'un équilibre existe, à un classement analogue à celui démontré en génétique mendélienne pour le coefficient de parenté.
\end{abstract}

\section{INTRODUCTION}

De récentes études (BrÉmont, Bouffetre et Boufferte, 1974) postulent que les mécanismes de la consanguinité impliquent des interactions entre des sites (ou gènes) des lots chromosomiques gamétiques du zygote. Le développement embryonnaire serait ainsi sous la dépendance d'un système répresseur-activateur (BIÉMONT, 1974) et dépendrait du nombre de couples de sites homologues hétéroactifs (un site actif, un site inactif). L'analyse mathématique de ce modèle a été réalisée dans le cas du système de croisement entre frère et sœur et aboutit à retrouver la plupart des effets expérimentaux de ce type de croisement (BIÉmont et al., 1974). Dans cet article, nous étudions l'évolution de ces interactions au sein d'une population sauvage, dans les conditions idéales de la panmixie.

Considérons l'évolution de l'état d'un site donné. Ce dernier peut être actif ou 
réprimé, lorsqu'on se rapporte à la théorie de " l'inbreeding " développée par BIÉMONT et BOUFFETTE (I974).

Il existe alors deux types gamétiques seulement, qui peuvent donner naissance à deux types zygotiques (et non trois comme le suppose la théorie mendélienne de 1'hérédité) : la fusion de deux gamètes réprimés donnant un individu adulte réprimé et tout autre croisement de gamètes donnant un adulte activé.

Il est peut-être ici utile de rappeler que, sauf modification au cours de la méiose, un adulte produit une unique catégorie de gamètes portant des sites dans le même état d'activation que ceux de l'adulte.

\section{I. - PANMIXIE}

Pour étudier, au niveau d'un site donné, une population panmictique, d'effectif illimité, nous utilisons la notion d'algèbre génétique. (ETHERINGTON, I940; BERTRAND, Ig66).

L'algèbre que nous proposons admet une base à deux éléments, A et R, correspondant respectivement aux deux génotypes possibles, zygote actif ou zygote réprimé.

Pour tenir compte des fréquences relatives des divers génotypes parmi les descendants des croisements entre zygotes de même type (ci-dessous, lignes I et 3 ) et entre zygotes de types différents (ci-dessous, ligne 2), nous avons muni l'algèbre de la loi de multiplication suivante ${ }^{(1)}$ :

$$
\left.\begin{array}{l}
\mathrm{AA}=\left(\mathrm{I}-\mu^{2}\right) \mathrm{A}+\mu^{2} \mathrm{R} \\
\mathrm{AR}=\mathrm{RA}=(\mathrm{I}-\mu+\mu \omega) \mathrm{A}+\mu(\mathrm{I}-\omega) \mathrm{R} \\
\mathrm{RR}=\left[\mathrm{I}-(\mathrm{I}-\omega)^{2}\right] \mathrm{A}+(\mathrm{I}-\omega)^{2} \mathrm{R}
\end{array}\right\}
$$

$\mu$ (respectivement $\omega$ ) représente la probabilité pour qu'un site d'un gamète soit actif (respectivement réprimé) sachant que ce site est réprimé (respectivement actif) chez l'adulte qui lui donne naissance.

Considérons alors une population nombreuse d'individus comprenant à la génération $n$ des proportions $p_{n}$ de type $\mathrm{A}$ et $q_{n}$ de type $\mathrm{R}$. Elle s'écrit symboliquement :

$$
\mathbf{P}=p_{n} \mathrm{~A}+q_{n} \mathrm{R}=\mathrm{A}+q_{n} \mathrm{~B} \quad \text { avec } \quad \mathrm{B}=\mathrm{R}-\mathrm{A} .
$$

Pratiquer un mode de croisement répété consiste donc à croiser au hasard deux individus ayant chacun la probabilité $p_{n}$ d'être $\mathrm{A}$ et $q_{n}$ d'être $\mathrm{R}$.

$$
\begin{aligned}
\left(p_{n} \mathrm{~A}+q_{n} \mathrm{R}\right)^{2} & =p_{n+1} \mathrm{~A}+q_{n+1} \mathrm{R} \\
\left(\mathrm{A}+q_{n} \mathrm{~B}\right)^{2} & =\mathrm{A}+q_{n+1} \mathrm{~B} \\
& =\mathrm{A}+\left(\mu+\gamma q_{n}\right)^{2} \mathrm{~B}
\end{aligned}
$$

expression obtenue en utilisant la multiplication définie par les formules ( $\mathbf{I}$ ) et en posant :

d'où :

$$
\gamma=\mathbf{I}-\mu-\omega
$$

$$
q_{n+1}=\left(\mu+\gamma q_{n}\right)^{2} .
$$

(1) Ces formules different de celles que l'on obtiendrait avec le modèle de dominance auquel pourrait penser le lecteur habitué au modèle classique. 
Cette équation de récurrence permet de déterminer, en fonction de la fréquence initiale $q_{0}$, la fréquence du type $\mathbf{R}$ au cours des générations successives.

\section{Étude du comportement asymptotique de la fréquence $q_{n}$.}

Si $\gamma$ est $n u l$, alors $q_{n}$ est égal à $\mu^{2}$ dès la première génération. La fréquence reste donc constante au cours du temps.

Supposons donc $\gamma$ non nul.

La suite récurrente $\left(q_{n}\right)$ est définie par :

$$
q_{n}=f\left(q_{n-1}\right)
$$

où $f$ est la fonction continue, définie de $(\mathrm{O}, \mathrm{I})$ dans $(\mathrm{O}, \mathrm{I})$ par :

$$
f(x)=(\mu+\gamma x)^{2} .
$$

Elle est croissante si $\gamma>0$, et décroissante si $\gamma<0$.

Lorsque $\gamma$ est positif, la suite $\left(q_{n}\right)$ est alors une suite monotone, bornée inférieurement par o et supérieurement par I, donc elle converge vers la limite $q$, solution comprise entre $o$ et I de l'équation :

$$
h(q)=(\mu+\gamma q)^{2}-q=0 .
$$

Une telle solution existe, puisque

Comme

$$
\begin{aligned}
h(0) \cdot h(\mathbf{I}) & =\mu^{2}\left[(\mathbf{I}-\omega)^{2}-\mathbf{I}\right] \leqslant 0 . \\
\gamma^{2} \cdot h(\mathbf{I}) & =\gamma^{2}\left[(\mathbf{I}-\omega)^{2}-\mathbf{I}\right] \leqslant 0,
\end{aligned}
$$

$q$ est la plus petite des deux racines de l'équation :

On a donc:

$$
(\mu+\gamma q)^{2}-q=0 .
$$

$$
q=\frac{\mathbf{I}-2 \mu \gamma-(\mathbf{I}-4 \mu \gamma)^{1 / 2}}{2 \gamma^{2}}
$$

Lorsque $\gamma$ est négatif, la fonction $f$ étant décroissante, il faut étudier les suites $\left(q_{3^{n}}\right)$ et $\left(q_{2^{n+1}}\right)$. Elles sont monotones, bornées, donc convergentes. Il est d'ailleurs facile de voir que si l'une de ces suites est croissante, l'autre est décroissante.

Les suites $\left(q_{2^{n}}\right)$ et $\left(q_{2^{n+1}}\right)$ sont alors définies à partir des relations suivantes :

$$
\begin{aligned}
q_{2^{n}} & =\left[\mu+\gamma\left(\mu+\gamma q_{2 n-2}\right)^{2}\right]^{2} \\
q_{2^{n+1}} & =\left[\mu+\gamma\left(\mu+\gamma q_{2 n-1}\right)^{2}\right]^{2} .
\end{aligned}
$$

Leurs limites $q^{\prime}$ et $q^{\prime \prime}$ sont des solutions, comprises entre o et $\mathrm{r}$, de l'équation :

$$
g(x)=\left[\mu+\gamma(\mu+\gamma x)^{2}\right]^{2}-x=0
$$

qui s'écrit aussi :

$$
g(x)=h(x) \cdot h(x)=0
$$


avec :

$$
\begin{aligned}
& h(x)=(\mu+\gamma x)^{2}-x \\
& k(x)=\gamma^{4} x^{2}+\gamma^{2}(\mathbf{I}+2 \mu \gamma) x+(\mathrm{I}+\mu \gamma)^{2} .
\end{aligned}
$$

$h(x)$ s'annule pour $x=q$.

$k(x)$ admet, lorsque l'expression $3+4 \mu \gamma$ est négative, deux racines réelles distinctes, $q_{1}$ et $q_{2}$, toutes deux comprises entre $o$ et $\mathrm{I}$.

$$
q_{1}=\frac{-(\mathrm{I}+2 \mu \gamma)+\sqrt{-3-4 \mu \gamma}}{2 \gamma^{2}} \quad q_{2}=\frac{-(\mathrm{I}+2 \mu \gamma)-\frac{\sqrt{-3-4 \mu \gamma}}{2 \gamma^{2}}}{2}
$$

Par suite, lorsque $3+4 \mu \gamma>0$, les suites $\left(q_{2 n}\right)$ et $\left(q_{2 n+1}\right)$ admettent la même limite $q$, ce qui signifie que la suite $\left(q_{n}\right)$ converge.

Par contre, si les suites $\left(q_{2^{n}}\right)$ et $\left(q_{\mathbf{2}^{n+1}}\right)$ admettent des limites distinctes $q^{\prime}$ et $q^{\prime \prime}$, ces dernières sont solutions du système :

d'où l'on tire :

$$
\begin{aligned}
& q^{\prime}=\left(\mu+\gamma q^{\prime \prime}\right)^{2} \\
& q^{\prime \prime}=\left(\mu+\gamma q^{\prime}\right)^{2}
\end{aligned}
$$

$$
q^{\prime}+q^{\prime \prime}=-\frac{\mathrm{I}+2 \mu \gamma}{\gamma^{2}} \quad \text { et } \quad q^{\prime} q^{\prime \prime}=\frac{(\mathrm{I}+\mu \gamma)^{2}}{\gamma^{4}}
$$

par suite, $q^{\prime}$ et $q^{\prime \prime}$ sont les racines réelles de l'équation :

$$
\gamma^{4} x^{2}+\gamma^{2}(\mathrm{I}+2 \mu \gamma) x+(\mathrm{I}+\mu \gamma)^{2}=0
$$

c'est-à-dire :

$$
k(x)=0 .
$$

Ainsi lorsque $q^{\prime}$ et $q^{\prime \prime}$ sont distinctes, elles sont égales à $q_{1}$ et $q_{2}$.

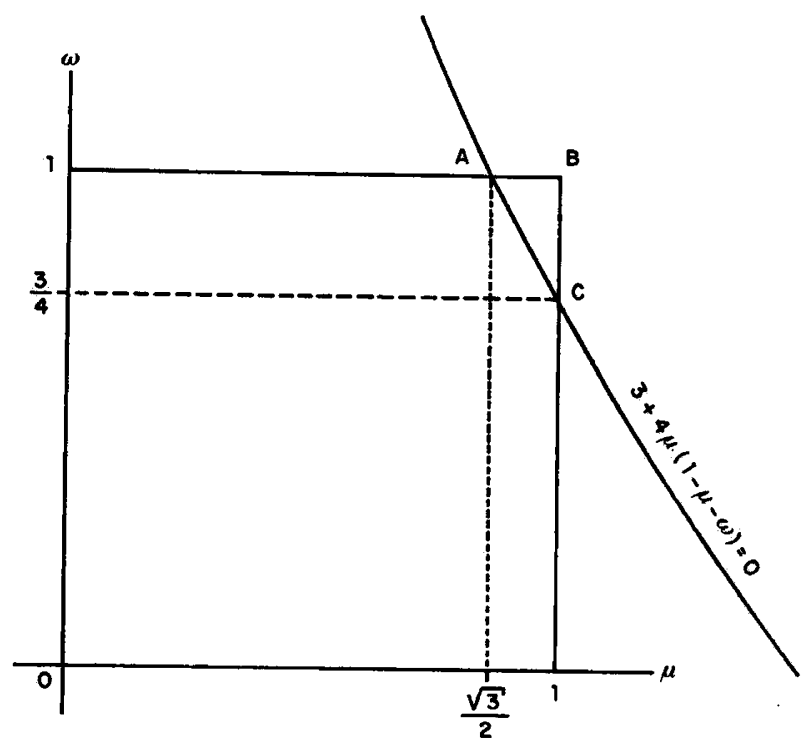

FIG. I. - Etude de la convergence de la suite $\left(q_{n}\right)$ 
Lorsque le temps augmente, la population tend vers une population stable dans laquelle il y a une proportion $q$ de type $\mathrm{R}$ et $\mathrm{I}-q$ de type $\mathrm{A}$, sauf lorsque les taux $\mu$ et $\omega$ sont suffisamment grands pour vérifier l'inégalité $3+4 \mu \gamma<0$. (Région $\mathrm{ABC}$ de la fig. I.) Dans ce dernier cas, la fréquence des types $\mathrm{R}$ oscillerait autour de deux valeurs $q^{\prime}$ et $q^{\prime \prime}$ : si elle est voisine de $q^{\prime}$ au cours d'une génération, elle sera voisine de $q^{\prime \prime}$ à la génération suivante, pour revenir au voisinage de $q^{\prime}$ lorsqu'on observera une nouvelle génération.

Il ne semble pas qu'un tel phénomène ait été observé. Peut-être a-t-on expliqué les écarts constatés par rapport à la loi de Hardy en faisant remarquer que les conditions expérimentales de la panmixie n'étaient pas parfaitement vérifiées et ainsi n'a-t-on pas poursuivi les observations?

A-t-on comparé les résultats obtenus pour les générations de rang pair entre eux d'une part, et ceux obtenus pour les générations de rang impair, d'autre part?

Il est cependant fort possible que des valeurs $q^{\prime}$ et $q^{\prime \prime}$ distinctes ne soient pas observées tout simplement parce que, dans la nature, ce cas ne se présentera pas : en effet cela demande des valeurs de $\mu$ et $\omega$ peut-être trop importantes (il est nécessaire mais non suffisant, que

$$
\left.\frac{\sqrt{3}}{2}<\mu \leqslant \mathrm{x} \quad \text { et } \quad \frac{3}{4}<\omega \leqslant \mathrm{I}\right) .
$$

\section{Remarque}

Lorsque $3+4 \mu \gamma$ est positif ou nul, alors la suite $\left(q_{n}\right)$ converge vers $q$, expression qui ne dépend pas de $q_{0}$. Or

$$
\begin{aligned}
q_{n+1}-q & =\left(\mu+\gamma q_{n}\right)^{2}-(\mu+\gamma q)^{2} \\
& =\gamma\left(q_{n}-q\right)\left[\left(\mu+\gamma q_{n}\right)+(\mu+\gamma q)\right]
\end{aligned}
$$

d'où, lorsque $\gamma>0,\left(q_{n}\right)$ est une suite monotone croissante ou décroissante suivant que $q_{0}$ est inférieur ou supérieur à la limite $q$. Par contre, si $-\frac{3}{4 \mu}<\gamma<0$, alors les suites $\left(q_{2 n}\right)$ et $\left(q_{2^{n+1}}\right)$ sont des suites adjacentes, telle que la suite $\left(q_{2^{n}}\right)$ croît ou décroît vers $q$ suivant que $q_{0}$ est inférieur ou supérieur à $q$.

\section{II. - COEFFICIENT DE DÉPRESSION ET PANMIXIE}

Le coefficient de dépression $D$ d'un individu est défini comme étant proportionnel au nombre moyen de couples homologues actifs et de couples homologues réprimés formés à partir des sites des deux gamètes qui lui donnent naissance; le coefficient de proportionnalité est égal à $\frac{\mathrm{I}}{\mathrm{N}}$, lorsque $\mathrm{N}$ désigne le nombre de sites étudiés.

Dans une population panmictique où $p_{n}$ est la probabilité pour qu'un site d'un individu adulte de la génération $n$ soit actif et $q_{n}$ celle pour qu'il soit réprimé, on obtient, en supposant que les différents sites aient tous la même probabilité d'être actifs, pour coefficient de dépression $D_{n_{+1}}$ d'un individu de la génération $n+\mathbf{I}$ :

$$
\begin{aligned}
& \mathrm{D}_{n+1}=\mathrm{I}-2\left[(\mathrm{I}-\mu) p_{n}+\omega q_{n}\right]\left[\mu p_{n}+(\mathrm{I}-\omega) q_{n}\right] \\
& \mathrm{D}_{n+1}=\mathrm{I}-2\left[(\mathrm{I}-\mu)-\gamma q_{n}\right]\left[\mu+\gamma q_{n}\right] \\
& \mathrm{D}_{n+1}=\mathrm{I}-2 \mu+2\left(q_{n+1}-\gamma q_{n}\right) .
\end{aligned}
$$


Si $\gamma=0$, alors $q_{n}$ est égal à $\mu^{2}$, donc :

$$
\mathrm{D}_{n+1}=\mu^{2}+(\mathrm{I}-\mu)^{2} \text {. }
$$

Le coefficient de dépression est invariant au cours du temps. Par contre, si $3+4 \mu \gamma \geqslant 0$, alors $D_{n}$ est fonction du temps, et tend, lorsque $n$ augmente indéfiniment, vers une limite $\mathrm{D}$ :

$$
\mathrm{D}=\mathrm{I}-2 \mu+2(\mu+\omega) q
$$

( $q$ désigne la solution comprise entre o et $I$, de l'équation $(\mu+\gamma q)^{2}=q$ ).

Si $3+4 \mu \gamma<0$, alors $D_{2 n}$ et $D_{2 n+1}$ tendent vers des limites distinctes $D^{\prime}$ et $\mathrm{D}^{\prime \prime}$ telles que :

$$
\begin{aligned}
& \mathrm{D}^{\prime}=\mathrm{I}-2 \mu+2\left(q^{\prime}-\gamma q^{\prime \prime}\right) \\
& \mathrm{D}^{\prime \prime}=\mathrm{I}-2 \mu+2\left(q^{\prime \prime}-\gamma q^{\prime}\right)
\end{aligned}
$$

expression dans lesquelles on a noté $q^{\prime}$ la limite de la suite $\left(q_{2^{n}}\right)$ et $q^{\prime \prime}$ celle de la suite $\left(q_{2 n+1}\right)$.

Remarquons que, dans ce dernier cas, les limites $D^{\prime}$ et $D^{\prime \prime}$ peuvent être égales sans que $q^{\prime}$ et $q^{\prime \prime}$ le soient. En effet, lorsque $\mu=\omega=I$, on a :

alors que :

$$
\left|\mathrm{D}^{\prime}-\mathrm{D}^{\prime \prime}\right|=2(\mathrm{I}+\gamma)\left|q^{\prime}-q^{\prime \prime}\right|=0
$$

$$
\left|q^{\prime}-q^{\prime \prime}\right|=\mathbf{I}
$$

D'ailleurs, dans ce cas, on a :

$$
\mathrm{D}^{\prime}=\mathrm{D}^{\prime \prime}=\mathbf{I}
$$

tandis que $q^{\prime}$ (ou $q^{\prime \prime}$ ) est nulle et $q^{\prime \prime}$ (ou $q^{\prime}$ ) vaut I.

\section{III. - CROISEMENTS PARTICULIERS}

\section{DANS UNE POPULATION PANMICTIQUE SUPPOSÉE A L'ÉQUILIBRE}

Les quatre sites homologues de deux couples d'adultes $M, L$ et $Q, P$ définissent I6 situations

$$
\mathrm{S}_{q p}^{m l}
$$

(BIÉmonr et al., I974). Si les adultes M, L, Q, P sont pris au hasard dans une population panmictique que l'on suppose être dans un état d'équilibre, la probabilité qu'un site de $\mathrm{M}$ (ou $\mathrm{L}$ ou $Q$ ou $\mathrm{P}$ ) soit réprimé est alors égale à $q$.

Par suite, en désignant par $v_{j}$ la probabilité pour qu'un quadruplet soit dans la situation $\mathbf{S}_{j}(j=\mathrm{I}, 2, \ldots, \mathrm{I} 6)$, on a :

$$
\begin{aligned}
& v_{1}=(\mathrm{I}-q)^{4} \\
& v_{2}=v_{3}=v_{4}=v_{5}=(\mathrm{I}-q)^{3} q \\
& v_{6}=v_{7}=v_{8}=v_{9}=v_{10}=v_{11}=(\mathrm{I}-q)^{2} q^{2} \\
& v_{12}=v_{18}=v_{14}=v_{15}=(\mathrm{I}-q) q^{3} \\
& v_{16}=q^{4} .
\end{aligned}
$$


Alors la comparaison entre le coefficient de dépression d'un individu issu de parents quelconques $D_{1}$, celui d'un individu issu de parents " demi-frère " $D_{2}$ et celui d'un individu issu d'un croisement " frère-sœur " $\mathrm{D}_{3}$ est immédiate (BIÉmONT et al., 1974).

On obtient, en considérant $\mathrm{N}$ quadruplets de sites homologues :

$$
\begin{aligned}
& \left(\mathrm{D}_{2}-\mathrm{D}_{1}\right)=2 \gamma^{4}\left\{\left[\mu v_{2}+(\mathrm{I}-\omega) v_{6}\right] \mu+\left[\mu v_{6}+(\mathrm{I}-\omega) v_{12}\right](\mathrm{I}-\omega)\right\} \\
& \left(\mathrm{D}_{3}-\mathrm{D}_{1}\right)=2 \gamma_{4}\left\{\mu^{2}\left(v_{2}+v_{6}\right)+(\mathrm{I}-\omega)^{2}\left(v_{6}+v_{12}\right)\right\}
\end{aligned}
$$

par suite :

$$
\mathrm{D}_{3} \geqslant \mathrm{D}_{\mathbf{2}} \geqslant \mathrm{D}_{\mathbf{1}} \text {. }
$$

Cet ordre est généralement celui que l'on observe dans les travaux expérimentaux.

\section{CONCLUSION}

Les hypothèses formulées par BIÉMoNT et BoufFETTE (I974) conduisent à des résultats théoriques comparables, pour la panmixie, à ceux obtenus en génétique mendélienne à l'aide du coefficient de consanguinité.

Ainsi, un classement différent des coefficients de dépression implique soit des valeurs particulières des paramètres $\mu$ et $\omega$ (afin que la limite $q$ n'existe pas), soit une structure non panmictique de la population; ce dernier cas est notamment rempli lorsque quelques individus seulement sont choisis pour réaliser les croisements consanguins.

Or la fréquence $q$ est uniquement fonction de $\mu$ et $\omega$. En supposant que ceux-ci sont caractéristiques de l'espèce, la population tend toujours vers le même équilibre lorsque le croisement se fait en panmixie avec un effectif suffisamment nombreux pour que la loi des grands nombres soit vérifiée. Dans ces conditions, une population démarrée à partir d'un seul couple doit, après avoir subi les effets des croisements consanguins dès les premières générations, atteindre un équilibre stable, identique à celui de la population sauvage d'origine du couple. Ces hypothèses ont pu être vérifiées expérimentalement par BrÉmont (1975).

Reçu pour publication en octobre 1975.

\section{SUMMARY}

\section{DEPRESSION COEFFICIENT AND RANDOM MATING}

According to the hypotheses advanced in the chromosomal inbreeding theory which imply gametic interactions controlling embryogenesis (BIEMONT, BoufFetTe, 1974), a random mating population can be characterized by an Etherington's algebra. The zygotic frequencies are calculated. A state of equilibrium for this interacting system is generally determined, which does not depend on the original structure of the founder population.

When equilibrium exists, the " coefficient de dépression" for random, half-sib and sib matings are ordered like the corresponding inbreeding coefficients calculated according to mendelian hypotheses.

However, when the parameter values of the model (the $\mu$ and $\omega$ " mutation " rates) are important, the zygotic frequencies and the "coefficient de dépression "may fluctuate between two limits. 


\section{RÉFÉRENCES BIBLIOGRAPHIQUES}

Bertrand M., x966. Algèbres non associatives et Algèbres génétiques. Memorial des Sciences Mathématiques, Fascicule CLXII. Paris, Gauthier-Villars éditeur.

Bremont C, Bouffetre A.-R., Bouffetre J., r974. Théorie chromosomique de l'inbreeding Modèle probabiliste. Bull. Math. Biol., 86, 4T7-434.

Bouffette A.-R., Bitmont C., Boufretre J., r975. Comparaison des croisements au hasard, frère-sœur et demi-frère-scur par le coefficient de dépression. Bull. Math. Biol., 37, 37-49.

Etherington I. M. H., r940. "Non-Associative Algebra and the Symbolism of Genetics Proc. Roy. Soc. Edinburgh, (B), 61, 24-42.

Malécot G., 1948. Les mathématiques de lhérédite. Masson, Paris. 